\title{
Jamming by compressing a system of granular crosses
}

\author{
Hu Zheng ${ }^{1,2, \star}$ Dong Wang ${ }^{2, \star \star}$ Jonathan Barés ${ }^{2,3, \star \star \star}$ Robert Behringer ${ }^{2, \star \star \star \star}$ \\ ${ }^{1}$ School of Earth Science and Engineering, Hohai University, Nanjing 210098, China \\ ${ }^{2}$ Department of Physics, Center for Nonlinear and Complex Systems, Duke University, Durham, NC, USA \\ ${ }^{3}$ Laboratoire de Mécanique et de Génie Civil de Montpellier, Université de Montpellier, France
}

\begin{abstract}
.
A disordered stress-free granular packing can be jammed, transformed into a mechanically rigid structure, by increasing the density of particles or by applying shear deformation. The jamming behavior of systems made of 2D circular discs has been investigated in detail, but very little is known about jamming for non-spherical particles, and particularly, non-convex particles. Here, we perform an experimental study on jamming by compression of a system of quasi-2D granular crosses made of photo-elastic crosses. We measure the pressure evolution during cyclic compression and decompression. The Jamming packing fraction of these quasi-2D granular crosses is $\phi_{J} \simeq 0.475$, which is much smaller than the value $\phi_{J} \simeq 0.84$ for- $2 \mathrm{D}$ granular disks. The packing fraction shifts systematically to higher values under compressive cycling, corresponding to systematic shifts in the stress-strain response curves. Associated with these shifts are rotations of the crosses, with minimal changes in their centers of mass.
\end{abstract}

\section{Introduction}

Granular materials can be easily found in nature and in industrial applications. They have gas-like, liquid-like or solid-like properties depending on their density or shear stress [1-4]. Many industrial devices exploit these state properties, and a number of geo-hazards involve uncontrolled state changes. For example, in controlled flow in an industrial process, such as in a hopper, gas-like or liquidlike states are desirable [5]. On the other hand, most geo-hazards, like landslide or debris flow occur when a jammed solid state transitions to a liquid-like state [6, 7]. Hence, it is very important to understand the granular phase properties, and the transitions between fluid and solid, i.e. unjammed or jammed, states.

Characterizing the jamming transition in disordered granular systems has attracted significant interest [2, 812]. Liu and Nagel [8] hypothesized a zero temperature jamming phase diagram. In a space of shear stress, $\tau$ and packing fraction, $\phi$, they proposed jammed states for $\tau<\tau_{Y}(\phi)$, where the yield stress curve, $\tau_{Y}(\phi)$, is a monotonically increasing curve that intersects the $\phi$ axis at $\phi_{J}$. This proposal was substantiated by O'Hern et al. using numerical simulations of frictionless discs. These studies used protocols that produced macroscopically isotropic states. Recently, Bi et al. [2] showed that for frictional disks, jammed states occur below the frictionless isotropic $\phi_{J}$. They used a protocol consisting of shear strain applied

\footnotetext{
^e-mail: hz64@phy.duke.edu

$\star \star$ e-mail: dw148@phy.edu.ed

$\star \star \star$ e-mail: jb@jonathan-bares.eu

$\star \star \star \star$ e-mail: bob@phy.duke.edu
}

to an initially unjammed (stress-free) isotropic state. In a range $\phi_{S}<\phi<\phi_{J}$, they found that increasing shear strain led from unjammed states to fragile and then robustly shear jammed states, where the last two states are characterized by non-zero shear stress. The isotropic jamming point of a two dimensional frictionless disc granular system is $\phi \simeq 0.84[9,13]$.

However, most of the experiments and simulations of jamming have focused on simple geometry particles like discs and spheres or at least on convex particles. Hence the focus of the present experiments is on non-convex crossshaped particles. Role of particle shape has been recognized as significant in controlling the microstucture of various granular materials [1, 14-16]. For instance, Athanassidis et al. [17] have shown that the macroscopic mechanical properties of a granular material can be controlled by tuning the grain shape via a 3D printing approach to make different shape particles including tetrahedra, octahedra, and dodecahedra[17]. And several authors have shown that 3D systems of rods [18] or of non-convex particles, such as staples, or star-shaped particles have interlocking or entangled states that are mechanically stable [1921]. For instance, star-shaped non-convex particles, i.e. hexapods are interesting to both granular science and architecture design. These particles form stable granular aggregates by pouring [22]. Zhao et al. [21] conducted laboratory experiments to show the stability and structure of poured hexapod packings by varying the arm length and coefficient of friction between particles. However, experimental methods generally cannot detect the internal details of such packings, or the forces between particles, which 


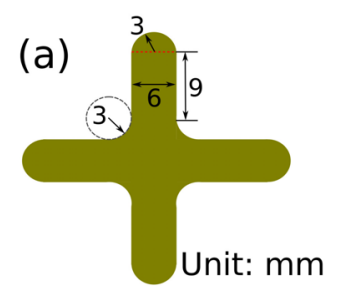

(c)

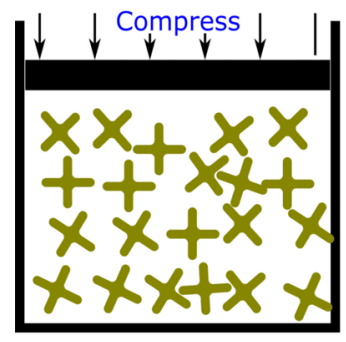

(b)
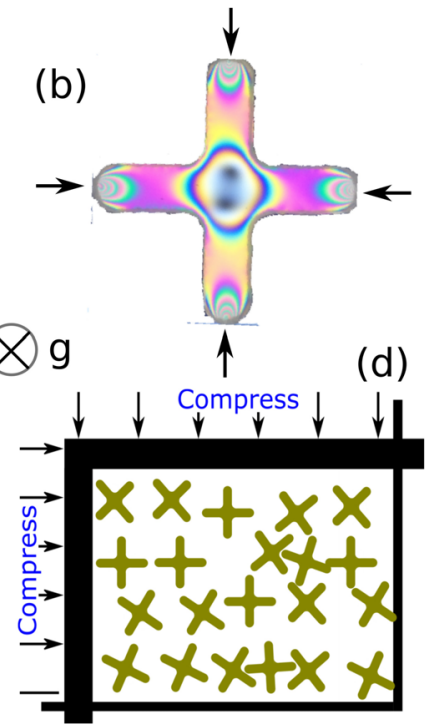

Figure 1. (a): Sketch of cross particle geometry. (b): Photoelastic response generated by compressing a cross particle along each arm. (c): Schematic of uniaxial compression, using the biaxial apparatus. (d): Sketch of biaxial compression. The gravity direction is given by $\mathbf{g}$.

are important for understanding their mechanical properties.

The photo-elastic method, used here, is an effective experimental technique to study 2D granular materials $[10,23-26]$ because it provides access to the forces, and hence local pressure on each particle. We combine this technique with direct visualiation of particle displacements and rotations. The latter is particulary important for crosses as they gradually evolve to lower stress states at a given $\phi$ under cyclic compression/decompression. We apply strain with a bi-axial apparatus $[2,27]$ which allows controlled strains in two directions, as in Fig. 1(c) and (d).

\section{Experimental techniques and protocols}

\subsection{Experimental techniques}

Specifically, the experiments are conducted using photoelastic crosses in a two dimensional bi-axial apparatus. We cyclically compress this system through the isotropic jamming point, and then decompress the system to the original boundary configuration. The geometry of the crosses, cut out of a $6.35 \mathrm{~mm}$ thick photo-elastic sheet by water-jet cutting, is shown in Fig. 1 (a). The length of each arm is $18 \mathrm{~cm}$, from the center of cross to the edge. The end of each arm is a semicircle and the corner between two neighbor arms is arc-shaped to avoid force concentration. The radii of the semicircle and arc is $3 \mathrm{~cm}$. The friction coefficient between crosses is $\sim 0.7$.

The particle photo-elastic property allows us to measure the forces acting on them via digital images of the

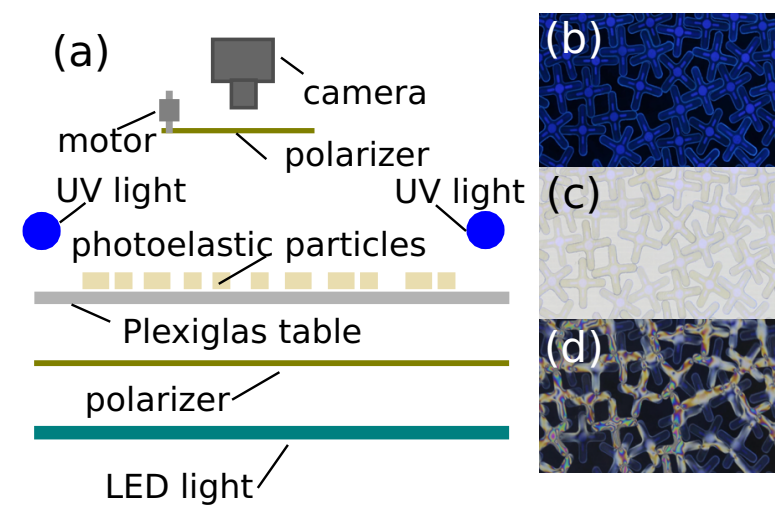

Figure 2. Experimental apparatus and typical experimental image data. (a): Schematic of the experimental apparatus, side view. Light from an LED source passes through a first circular polarizer, then through the transparent base of the apparatus and the particles. A stepper motor rotates a polarizer into or out of the line of sight of the camera, yielding polarized or non-polarized images. (b): Image with only UV light from above. Fluorescent bars on the arms yield the cross orientation. (c): Image with normal light and without the second polarizer. These images are used to detect particle positions. (d): Polarized image used to measure the pressure inside of the each particle.

fringe patterns, as typified by Fig. 1 (b). In particular, the concentrated intensity variation close to a contact point gives a measure of the force at a contact, and helps determine the existence of contacts. The density of fringes corresponds to the magnitude of the force: the more fringes, the bigger the force. Fig. 1 (b) shows a photo-elastic image of a cross particle subjected to compression in all four directions, as indicated by the arrows. We use the ' $G^{2}$ ', method [23] as measure of the fringe density, and hence of the forces acting on a particle. The $G^{2}$ value is defined as the pixel-wise intensity gradient in the photo-elastic digital image. Moreover, the actual contact force can also be calculated in principle based on a complex fringe patternfitting algorithm pioneered by Majmudar [27]. In this paper, we only use $G^{2}$ as a measure of the pressure of each particle, which has been demonstrated to be a one-to-one function of pressure at the particle scale [28].

\subsection{Experimental apparatus}

The experimental apparatus performed bi-axial and uniaxial compression. An illustration of the system is shown in Fig 2 (a). The photo-elastic cross particles are placed on a transparent Plexiglas table in a horizontal plane, such that gravity plays no role in the granular behavior, other than through weak friction with the base $(0.36 \pm 0.04)$. Four UV tube lights illuminate the UV-fluorescent ink on the top of each cross (Fig. 2 (b)), allowing us to track the position and orientation of each cross. An LED array provides homogeneous light from below. A circular polarizer sheet on the top of the LED array polarizes the light. A second crossed polarizer is rotated into or out of position in front of a camera, providing normal and polarized images (Fig. 2 (c) and (d)). The camera (Canon $\left.{ }^{\circledR} 70 D\right)$ 
has a resolution of $3648 \times 5472$ pixels, corresponding to $\sim 31,000$ pix $^{2}$ per cross.

\subsection{Protocol}

We compressed the system quasi-statically in steps of $1 \mathrm{~mm}$. After each step, we obtained three images, one with only UV light, one polarized and a normal image with light from the LED array. The initial size of the shear box was $47.2 \times 39.3 \mathrm{~cm}^{2} .270$ crosses were placed in the shear box. Before compressing the system, we ensured that there was no initial stress in the system, which meant that no fringes were visible in the polarized image of the initial state.

\section{Results and discussion}

We focus on the jamming transition where we contrast system properties that were achieved by compressing either uniaxially or isotropically, i.e. biaxially. Below, we distinguish these two protocols by unicompression or bicompression. For these two protocols, we determined the contact number, the force chain network, and the system pressure, and used these properties to characterize the jamming transition.

The system pressure $\left(G^{2}\right)$ evolution as a function of the packing fraction for multiple compression cycles is shown in Fig. 3. The blue line corresponds to unicompression and the red one to bicompression. The green region indicates the transition area of the granular crosses from an unjammed to a jammed state. Manifestly, the jamming point is not a unique function of packing fraction, and it also depends on the compression protocol. The difference between unicompression and bicompression can be seen from the inset images in Fig. 3. The force chain network generated by unicompressing is more anisotropic than the

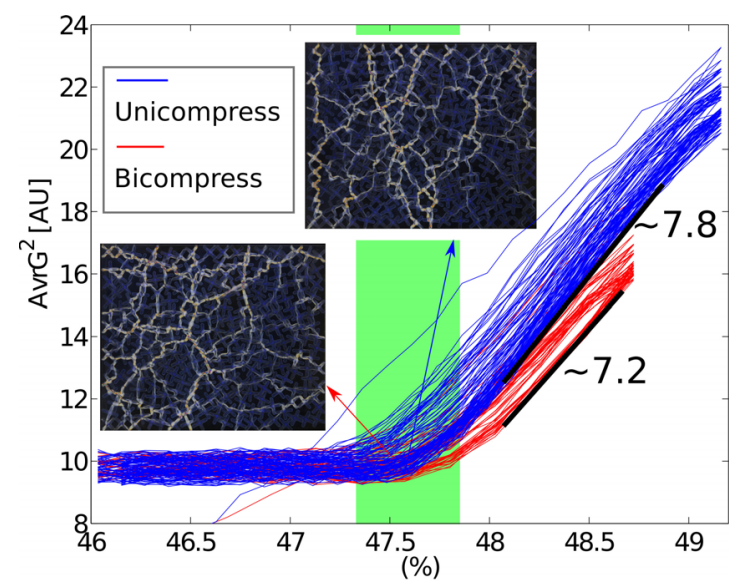

Figure 3. System pressure, expressed by $G^{2}$ in arbitrary units, $v s$. compressive strain for multiple cycles. Insets: Image indicated by red arrow is a photo-elastic image at the jamming point for unicompressing while the one indicated by blue arrow is for bicompression. The force chain networks of the two images indicate that a more isotropic network occurs for the bicompression case. The green area shows the jamming transition zone.

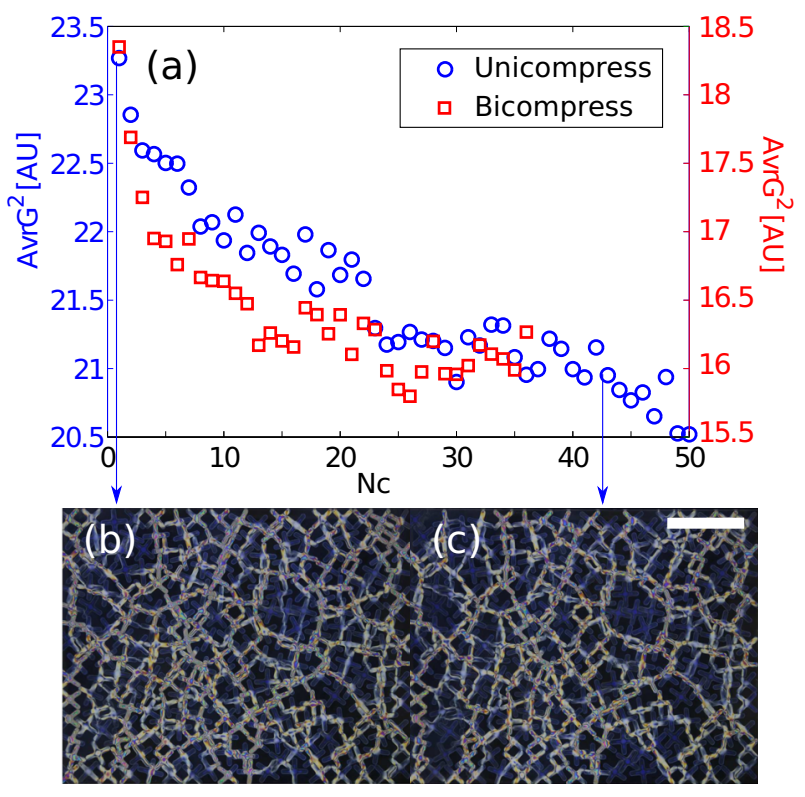

Figure 4. (a): Maximum $G^{2}$ at the highest packing fraction of each compression circle. Note that the maximum compressions and vertical scales are different for the two compression protocols. The maximum $G^{2}$ decays with $N_{c}$, the number of compression cycles for both experimental methods. Alternatively, this can be seen as an increase in $\phi_{J}$ as a function of $N_{c}$. (b): Photo-elastic image when the system firstly reached the maximum packing fraction, $\phi_{\max }=48 \%$. (c): Photo-elastic image at the maximum packing fraction after 43 shearing cycles. The white bar in (b) indicates $10 \mathrm{~cm}$. (b) and (c) share the same scale.

bicompression case, reflecting the more anisotropic character of unicompression. The difficulty of rearrangement in the cross packing also caused a relative fast $G^{2}$ development by unicompressing with a slope of about 7.8. For the bicompression system, the slope of $G^{2}$ is about 7.2, and the system evolution is smoother.

The crosses move little during cyclic compression, $G^{2}$ decays over multiple cycles, as seen in 2D disc system [13] and in simulations of frictionless spheres. However, $G^{2}$ appears to reach a plateau, within moderate fluctuations, for large enough $N_{c}$ for bicompression, although not necessarily for unicompressions. Fig. 4 (b) and (c) show the force chain networks at the packing fraction, $\phi=48 \%$. The photo-elastic response at cycle 43 is slightly weaker than after the first cycle.

We compared the position and orientation of each cross in the unicompression system, as shown in Fig. 5, to see that the position of each particle changes very little, even after 43 compression cycles; consequently, the coarse-grain density also changes little over the 43 cycles. However, the particle orientations change substantially. It is reasonable to conclude that the pressure decay at the maximum packing fraction is strongly correlated with changes in the particle orientations. 


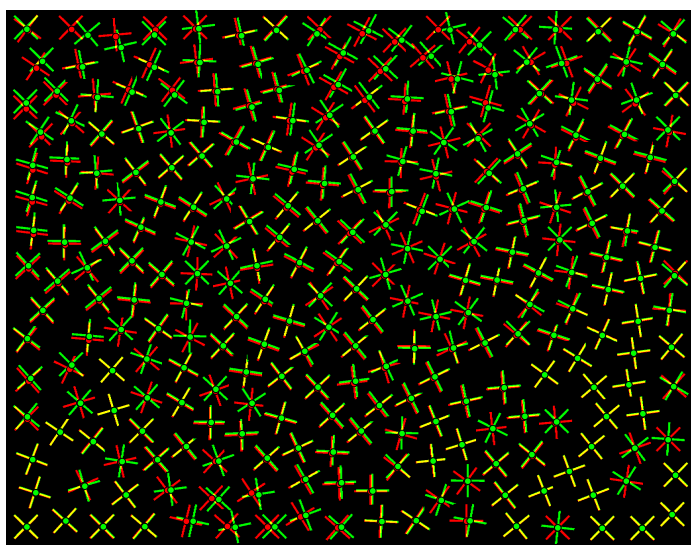

Figure 5. Positions and orientations of the crosses for unicompression initially (red crosses) and after 43 compression cycles (green crosses).

\section{Conclusion}

We have experimentally investigated the jamming transition of a packing formed from 2D photoelastic crosses subject to multiple cycles of compression that is either uniaxial or biaxial. The jamming point of this system is between $47.5 \%$ and $48.0 \%$, and the specific jamming density is sensitive to details of the compression protocol, including the cycle number and whether the compression is unixial of biaxial. The rate of increase of $G^{2}$ with $\phi$ is higher for unicompression than for bicompression. Several measures are sensitive to the cycle number, including the jamming point and the pressure at a given packing fraction two properties that are correlated. There is little change in the positions of individual particles, but there is significant rotation of crosses during the course of multiple cycles, and this rotation provides a mechanism for stress relaxation.

\section{Acknowledgements}

This work is supported by NSF Grant No.DMR1206351, NASA Grant No. NNX15AD38G, the W.M. Keck Foundation and a RT-MRSEC fellowship. HZ thanks NSFC Grant No. 41672256 and the NSF(Jiangsu, China) Grant No. BK20140845 for financial support.

\section{References}

[1] H.M. Jaeger, S.R. Nagel, R.P. Behringer, Reviews of Modern Physics 68, 1259 (1996)

[2] D. Bi, J. Zhang, B. Chakraborty, R.P. Behringer, Nature 480, 355 (2011)

[3] M. van Hecke, Journal of Physics: Condensed Matter 22, 033101 (2010)

[4] Alexander O.N. Siemens, Martin van Hecke, Physica A 389, 4255 (2010)
[5] J. Tang, R.P. Behringer, Europhysics Letters 114, 34002 (2016)

[6] G.Q. Chen, R.Q. Huang, Q. Xu, T. Li, M. Zhu, Journal of Mountain Science 10, 532 (2013)

[7] H. Zheng, Y. Huang, Environmental Earth Sciences 73, 4853 (2015)

[8] A.J. Liu, S.R. Nagel, Nature 396, 21 (1998)

[9] C.S. O’Hern, L.E. Silbert, A.J. Liu, S.R. Nagel, PHYSICAL REVIEW E 68, 011306 (2003)

[10] R. Behringer, D. Bi, B. Chakraborty, S. Henkes, R.R.Hartley, Physical Review Letters 101, 268301 (2008)

[11] J. Ren, J.A. Dijksman, R.P. Behringer, Physical Review Letters 110, 018302 (2013)

[12] H. Zheng, J.A. Dijksman, R.P. Behringer, Europhysics Letters 107, 34005 (2014)

[13] T. Majmudar, M. Sperl, S. Luding, R. Behringer, Physical review letters 95, 058001 (2007)

[14] J. Duran, Sands, powdersm and Grains: An Introduction to Physics of Granular Materials (Springer, Berlin, 1999)

[15] D.M. Wood, Soil Behavior and Critical State Soil Mechanics (Cambridge University Press, Cambridge, 1990)

[16] E. Azéma, F. Radjaï, R. Peyroux, G. Saussine, Physical Review E 76, 011301 (2007)

[17] A. Athanassiadis, M. Miskin, P. Kaplan, N. Rodenberg, S. Lee, J. Merritt, E. Brown, J. Amend, H. Lipson, H. Jaeger, Soft Matter 10, 48 (201)

[18] K. Desmond, S.V. Franklin, PHYSICAL REVIEW E 73, 031306 (2006)

[19] C. Schreck, N. Xu, O’HernaC.S., Soft Matter 6, 2960 (2010)

[20] T. Marschall, S. Franklin, S. Teitel, Granular Matter 17, 121 (2015)

[21] Y. Zhao, Kevin Liu, Matthew Zheng, Jonathan Barés, Karola Dierichs, Achim Menges, Robert P. Behringer, Granular Matter 18, 1 (2016)

[22] K. Dierichs, A. Menges, Architectural Design 85, 86 (2015)

[23] D. Howell, R.P. Behringer, C. Veje, Physical Review Letter 82, 5241 (1999)

[24] J. Geng, D. Howell, E. Longhi, R.P. Behringer, G. Reydellet, L. Vanel, E. Clément, S. Luding, Physical Review Letter 87, 035506 (2001)

[25] A.H. Clark, R.P. Behringer, Europhysics Letters 101, 64001 (2013)

[26] A.H. Clark, A.J. Petersen, R.P. Behringer, Physical Review E 89, 012201 (2014)

[27] T.S. Majmudar, R.P. Behringer, Nature 435, 1079 (2005)

[28] M. Cox, D. Wang, J. Barés, R.P. Behringer, Europhysics Letters 115, 64003 (2016) 